\title{
Tomographic Reconstruction for Truncated Cone Beam Data Using Prior CT Information
}

\author{
Krishnakumar Ramamurthi and Jerry Prince \\ Johns Hopkins University, Electrical and Computer Engineering, \\ Baltimore, MD 21218, USA \\ $\{$ krishna,prince $\} @ j h u . e d u$
}

\begin{abstract}
C-arms can be used in surgery to acquire rotational conebeam data, which can be used to obtain three-dimensional reconstructions of anatomy. In many scenarios, there is insufficient space or capability to obtain the large angular rotations required to reconstruct artifact-free anatomy. Projection data can also suffer from truncation, which causes additional artifacts in three-dimensional reconstruction. In this paper we present a method that compensates for truncation using prior information from computed tomography and provides accurate reconstruction in the mid-plane under certain conditions.
\end{abstract}

\section{Introduction}

Demand for three-dimensional (3D) imaging during surgery is growing. This capability is particularly important for pelvic surgeries, where precise visualization and mensuration is important for catheter, endoscope, and biopsy needle placement and for precise alignment of bony structures in hip replacement and other pelvic surgeries. While pre-operative computed tomography (CT) data might be commonly available, articulation and deformation of the pelvis structures make precise intra-operative registration and planning impossible without intra-operative imaging.

Use of fluoroscopic C-arms is an attractive option for three-dimensional (3D) reconstruction during surgery because $\mathrm{C}$-arms are often found in the OR, are cheaper and more flexible than CT scanners, and they can be used in both the generation of volume images and real-time 2D projection images. Yet limitations in both the total sweep angle and field-of-view (FOV) that are practical for a $\mathrm{C}$-arm in pelvic surgeries make it difficult, if not impossible, to produce accurate, artifact-free volumetric reconstruction from $\mathrm{C}$-arms today.

In this paper, we consider a restricted scenario for volumetric reconstruction from C-arms in surgery. We assume that a prior CT scan is available and that the 2D C-arm projections can be accurately registered to the pre-operative CT scan. We further assume that the surgery itself only affects a relatively small volume which we shall refer to as a region of change (ROC). While it is assumed that the $\mathrm{C}$-arm projections capture the ROC completely in all its views they are otherwise allowed to be truncated. Finally, we assume that the convex hull of the C-arm source path contains the entire projection of the ROC onto the mid-plane (the 
plane of the source path). For this scenario, which may apply to a wide variety of surgeries (especially of the minimally-invasive variety), we are able to achieve (using simulated projection data) high quality volume reconstructions of the entire object contained within the pre-operative $\mathrm{CT}$ scan. Remaining practical issues that must be addressed include 2D-3D registration, geometric corrections, and x-ray energy differences between the intra-operative projection images and the pre-operative CT scan.

\section{Background}

In the past 15 years, many cone beam reconstruction algorithms have been proposed. These can be broadly classified into two classes: algorithms that apply to source geometries allowing perfect reconstruction and algorithms that permit perfect reconstruction only in a central plane through the object. The condition for perfect reconstruction (Tuy's condition) [1/2] is hard to satisfy in practice. It requires that every plane through the object support intersect the source orbit. Given our application in surgery, this is almost never possible.

The second class of algorithms apply to a circular source path. For this geometry, the algorithm proposed by Feldkamp, Davis, and Kress (FDK) [3] is commonly used. This algorithm adapts the fan-beam filtered back-projection (FBP) algorithm, typically used in planar CT scanners [4], to three dimensions. In fact, it reduces to a fan-beam algorithm in the mid-plane, i.e., the plane containing the circular source path. Therefore, provided that projections are obtained over at least an angular range of $180^{\circ}+$ the cone angle (a so-called short-scan), Parker weights are used to account for redundant data [5], and no projection truncation occurs, FDK yields perfect reconstruction in the midplane. Off the mid-plane (in the $z$-direction), however, it is not perfect, and some undesirable artifacts appear with increasing severity with increasing distance from the mid-plane.

Patient and operating room (OR) setup further compound the problem of using the FDK algorithm for C-arm applications in surgery. For instance, consider the task of reconstruction from a set of heavily truncated projections obtained over only $180^{\circ}$ (less than a short scan). Fig. 1a shows a simulation of one such projection, that might be produced during a hip replacement surgery, for example. Fig. 1b shows a mid-plane FDK reconstruction resulting from a collection of such truncated projections over $180^{\circ}$. For comparison, Fig. 1c shows what the slice should look like. The combination of truncation artifacts and an inadequate angular sweep produces a variety of undesirable artifacts, which only get worse for slices farther away from the mid-plane.

This paper addresses both limited-angle scanning and truncation in threedimensional cone-beam reconstruction. The key idea is that prior CT data can be applied where no surgical changes have taken place and an optimized conebeam reconstruction can be used to reconstruct the ROC. It follows from noting that Tuy's condition says that it is possible to reconstruct an object lying within the convex hull of the source path. A recent result in fan-beam tomography by Noo, Defrise et al. [6] gives an exact reconstruction algorithm for this scenario. 


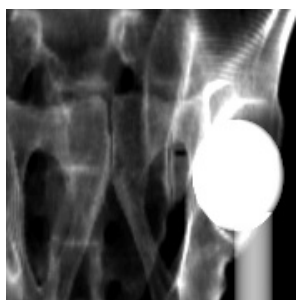

(a)

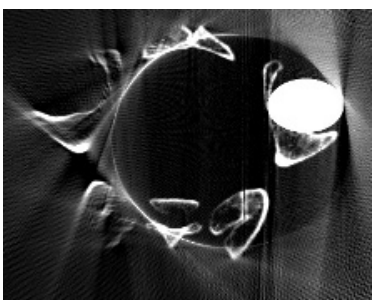

(b)

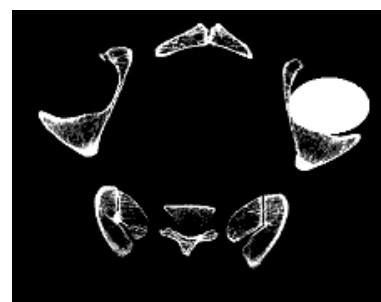

(c)

Fig. 1. (a) Simulated truncated projection; (b) an FDK mid-plane reconstruction from a $180^{\circ}$ scan; and (c) the true mid-plane image.

Unfortunately, both the Tuy's condition and the Noo algorithm require nontruncated projections, which we do not have. Furthermore, the Noo algorithm does not address how to reconstruct slices lying off the mid-plane.

In this paper, we make two contributions that lead to a fairly dramatic improvement in the reconstructions for this scenario by imposing certain requirements. First, we use the prior CT data to yield truncation-free projections so that the Noo algorithm can be used to get a perfect reconstruction within the convex hull of the source path. Second, we use an FDK-like extension to 3D in order to generate a reconstruction over the whole volume. Like FDK, our reconstruction is not optimal over the whole volume (unlike the mid-plane), but it is computationally fast enough to be practical for volumetric reconstruction.

\section{Theory}

\subsection{Two-Dimensional Tomographic Reconstruction}

Here we present some standard definitions and results in conventional twodimensional tomographic reconstruction [4]. The two-dimensional Radon transform of $f(x, y)$ is given by,

$$
\mathcal{P}_{f}(\ell, \theta)=\int_{-\infty}^{+\infty} \int_{-\infty}^{+\infty} f(x, y) \delta(\ell-x \cos \theta-y \sin \theta) d x d y .
$$

This transformation gives the line integral of $f(x, y)$ on a line parameterized by $\ell$ and $\theta$, as shown in Fig. 2a. The inverse 2-D Radon transform is given by

$$
f(x, y)=\int_{0}^{\pi}\left[\mathcal{P}_{f}(\ell, \theta) * \operatorname{ramp}(\ell)\right]_{\ell=x \cos \theta+y \sin \theta} d \theta,
$$

where $*$ represents one-dimensional (1D) convolution and

$$
\operatorname{ramp}(\ell)=\int_{-\infty}^{+\infty}|\nu| e^{j 2 \pi \nu \ell} d \nu
$$


In practice, line integrals are usually measured over a family of diverging lines known as a fan-beam. For every view angle $\beta$, projections are obtained in a fan emanating from the corresponding source position $\mathbf{S}(\beta)$ (Fig. 2b). The projections are parameterized by the variable $t$ on a virtual detector (for theoretical convenience) that passes through the origin and is perpendicular to the central ray $\overline{\mathbf{S O}}$.

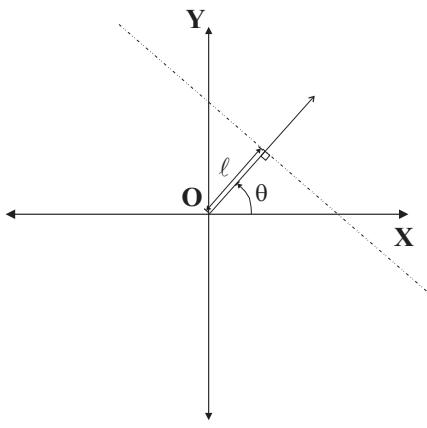

(a)

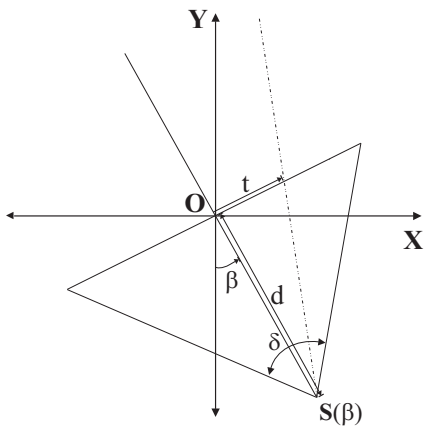

(b)

Fig. 2. (a) 2D Radon Transform: $(\ell, \theta)$ parameterizes a line; (b) Fan Beam Geometry: $\delta$ is the fan angle, $d$ is the focal-length and $(t, \beta)$ parameterizes a line

Let $\mathcal{D}_{f}(t, \beta)$ denote the line integral of the function $f(x, y)$ on the line parameterized by $t$ and $\beta$. It can be shown from the geometry (Figs. 2a and 2b) that,

$$
\mathcal{P}_{f}(\ell, \theta)=\mathcal{D}_{f}\left(t^{*}, \beta^{*}\right)
$$

where,

$$
t^{*}=\frac{\ell d}{\sqrt{d^{2}-\ell^{2}}} \quad \text { and } \quad \beta^{*}=\theta+\cos ^{-1} \frac{\sqrt{d^{2}-\ell^{2}}}{d} \operatorname{sgn}(\ell)
$$

A fan-beam reconstruction formula can be derived by applying the change of variables in (5) to (2) and introducing Parker weights [5] to handle redundancy [4]. This yields a fan-beam reconstruction formula that requires an angular sweep of at least $\pi+\delta$ radians, i.e., a short scan.

\subsection{Very Short Scan Fan-Beam Reconstruction}

Recent work by Noo, Defrise et al. [6] shows that with non-truncated fan-beam projections, one can perfectly reconstruct regions that lie in the convex hull of the source path. In particular, it is shown that (using our notation)

$$
\left|\frac{d \theta}{d \beta}\right|\left[\mathcal{P}_{f}(\ell, \theta) * \operatorname{ramp}(\ell)\right]_{\ell=x \cos \theta+y \sin \theta}=\frac{\sqrt{d^{2}+t^{* 2}}}{2 \pi d\left\|\mathbf{x}-\mathbf{S}\left(\beta^{*}\right)\right\|} \mathcal{D}_{F}\left(t^{*}, \beta^{*}\right),
$$

where $\mathbf{x}=(x, y)$, and the filtered projection is given by 


$$
\mathcal{D}_{F}(t, \beta)=\mathcal{H}_{t}\left[\frac{d}{\sqrt{d^{2}+t^{2}}}\left(\frac{\partial}{\partial \beta}+\frac{d^{2}+t^{2}}{d} \frac{\partial}{\partial t}\right) \mathcal{D}_{f}(t, \beta)\right],
$$

and $\mathcal{H}_{t}[\cdot]$ is the Hilbert transform operator with respect to $t$. Equation (6) allows us to compute the integral in (2) over $\beta$, while $t$ becomes a function of $x, y$, and $\beta$. It follows from Eqs. (2), (5), and (6) that

$$
f(x, y)=\left.\int_{\cos ^{-1} \frac{\sqrt{d^{2}-x^{2}}}{d} \operatorname{sgn}(x)}^{\pi-\cos ^{-1} \frac{\sqrt{d^{2}-x^{2}}}{d} \operatorname{sgn}(x)} \frac{1}{2 \pi\|\mathbf{x}-\mathbf{S}(\beta)\|} \frac{\sqrt{d^{2}+t^{2}}}{d} \mathcal{D}_{F}(t, \beta)\right|_{t=t^{*}(x, y, \beta)} d \beta .
$$

It can be shown geometrically that when projections are obtained over the range given by the limits of the integral in (8), the point $(x, y)$ lies exactly on the boundary of the convex hull of the source path. In general, for any given sweep $(\beta \in(0, \gamma))$ of a source path, some points may also lie inside the boundary of the convex hull. In this case there is excess or redundant information in the fan-beam data set. An appropriate weighting function should be used 6] to handle redundancy, and provide a unified reconstruction result for points that are contained in the convex hull of the source path:

$$
f(x, y)=\left.\frac{1}{2 \pi} \int_{0}^{\gamma} \frac{1}{\|\mathbf{x}-\mathbf{S}(\beta)\|} \frac{\sqrt{d^{2}+t^{2}}}{d} \mathcal{D}_{F}(t, \beta) w(t, \beta)\right|_{t=t^{*}(x, y, \beta)} d \beta
$$

where the weighting function $w(t, \beta)$ is defined in 6]. It can be shown by geometric arguments that for a circular source path and a finite size detector, a minimum sweep of $\pi-\delta$ radians is required to guarantee the inclusion of any part of the FOV inside the convex hull of the source path. Therefore, this reconstruction algorithm applies when the sweep angle is in the range $\pi \pm \delta$.

\section{Methods}

In this section we address the task of three-dimensional reconstruction from truncated cone-beam projections. It is assumed that a registered pre-operative CT scan is available, and that surgery has affected only a small region of change (ROC). We also assume that this ROC is always in the field of view (FOV) of the projections, i.e., not truncated as shown in Fig. 3a. We present a method that both compensates for truncation using the prior CT and adapts the very short scan fan-beam algorithm to cone-beam geometry.

\subsection{Cone-Beam Geometry}

The cone-beam geometry as shown in Fig. 3b consists of a single source $\mathbf{S}$ that projects a $3 \mathrm{D}$ object onto a $2 \mathrm{D}$ detector plane (st plane). The source-detector combination revolves about the $Z$ axis, given by the angle $\beta$ that $\overline{\mathbf{S O}}$ makes with the negative $Y$ axis. Let $\mathcal{D}_{f}(s, t, \beta)$ denote the line integral of $f(\mathbf{x})$ measured along the line joining the source to the point $(s, t)$ on the detector, where $f(\mathbf{x})$ represents the $3 \mathrm{D}$ object being imaged. 


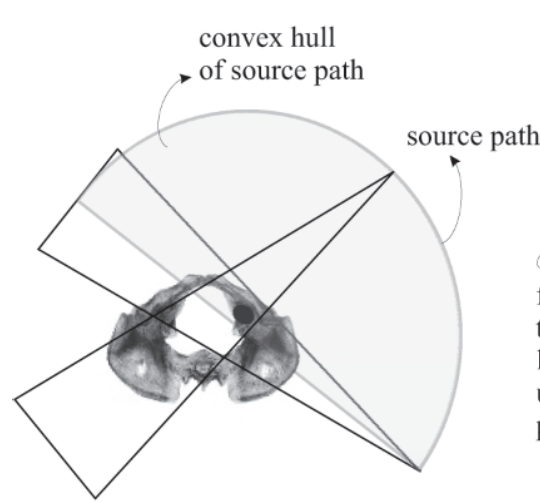

(a)

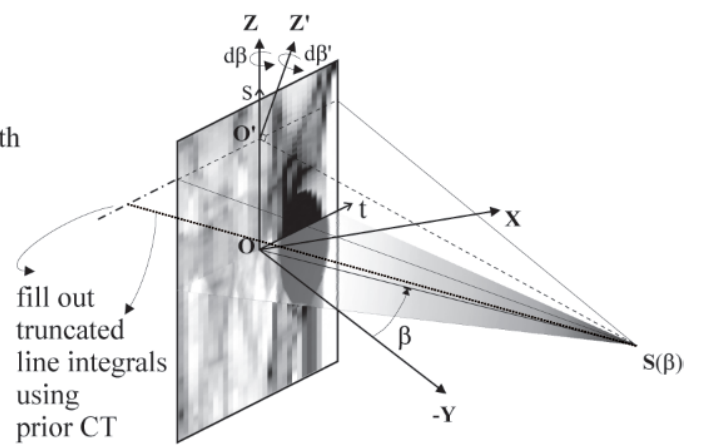

(b)

Fig. 3. (a) The elliptical black portion represents the region of change (ROC). It is assumed that the ROC is always in the FOV and hence not truncated (b) Cone-beam geometry may be treated as a stack of fan-beam geometry with varying focal lengths $\left(d=|\mathbf{S O}|\right.$ and $\left.d^{\prime}=\left|\mathbf{S O}^{\prime}\right|\right)$. The individual rows are filtered in accordance with (7) after compensating for truncation using a prior $\mathrm{CT}$

\subsection{Regional Reconstruction}

In an approach similar to that of the FDK algorithm [3], we treat the rows of the cone-beam projection as a stack of $1 \mathrm{D}$ fan-beam projections (Fig. 3b). We first compensate (where necessary) for truncation of data along these rows by reprojecting the prior CT along lines originating from the source to the points that lie outside the detector's extent. Let $\tilde{\mathcal{D}}_{f}(s, t, \beta)$ denote the truncationcompensated cone-beam projection that encompasses the whole object.

For any given row (fixed $s$ ), we have a fan-beam projection $\tilde{\mathcal{D}}_{f}(s ; t, \beta)$ that has a focal-length $d^{\prime}$ and rotates incrementally $\left(d \beta^{\prime}=d \beta \frac{d}{d^{\prime}}\right)$ about the $Z^{\prime}$ axis as the source rotates incrementally $(d \beta)$ about the $Z$ axis (see Fig.3b). We apply Noo's algorithm to these tilted fans and integrate the incremental contributions from them to obtain a back-projected volume that includes the ROC

$$
f(\mathbf{x})=\left.\frac{1}{2 \pi} \int_{0}^{\gamma} \frac{d}{\|\mathbf{x}-\mathbf{S}(\beta)\|} \frac{\sqrt{d^{2}+s^{2}+t^{2}}}{d^{2}+s^{2}} \tilde{\mathcal{D}}_{F}(s, t, \beta) w(t, \beta)\right|_{s=s^{*}(\mathbf{x}, \beta), t=t^{*}(\mathbf{x}, \beta)} d \beta,
$$

where $\left(s^{*}(\mathbf{x}, \beta), t^{*}(\mathbf{x}, \beta)\right)$ is the point where the line joining $\mathbf{S}(\beta)$ and $\mathbf{x}$ intersects the detector. The filtered projection in (10) is given by

$$
\tilde{\mathcal{D}}_{F}(s, t, \beta)=\mathcal{H}_{t}\left[\frac{\sqrt{d^{2}+s^{2}}}{\sqrt{d^{2}+s^{2}+t^{2}}}\left(\frac{\partial}{\partial \beta}+\frac{d^{2}+s^{2}+t^{2}}{\sqrt{d^{2}+s^{2}}} \frac{\partial}{\partial t}\right) \tilde{\mathcal{D}}_{f}(s, t, \beta)\right]
$$

and $\gamma \in(\pi-\delta, \pi+\delta)$ is selected so that the the projection of the ROC onto the mid-plane lies in the convex hull of the source path (Fig. 3a). 
Our final 3D volume result is arrived at by using the filtered back-projection steps (Eqs. (10) and (11)) only for those points $\mathbf{x}$, that when projected onto the mid-plane $(z=0)$, lie inside the convex hull of the source path. For all other points, we retain the prior CT values. Since the cone-beam geometry is a fanbeam in the mid-plane [3], it follows (from Sect.3] that our method reconstructs the ROC perfectly in the mid-plane.

\section{Results and Discussion}

The results of this simulation study are shown in Fig. 4 and described in its captions. A pelvic CT data set, serves as our prior CT information. To this, we add a mathematical phantom (ellipse and cylinder) that imitates a ball and socket implant to obtain a modified CT. This modified CT is used solely for the purpose of simulating truncated cone-beam projections $\left(180^{\circ}\right.$, one every degree). The ball and socket implant defines our ROC. The cone-beam projections were obtained using a cone-beam simulator called Take [7] with a cone-angle of $(\delta=$ $15^{\circ}$ ). For the implementation of our method, $\gamma$ was set to $175^{\circ}$ in (10) so as to include the projection of the ROC onto the mid-plane, inside the convex hull of this source path. While the filtering steps were implemented using fast Fourier space methods using FFT, back-projection steps were efficiently implemented by using projection matrices that intrinsically describe the projection geometry [8], [9] and [10].
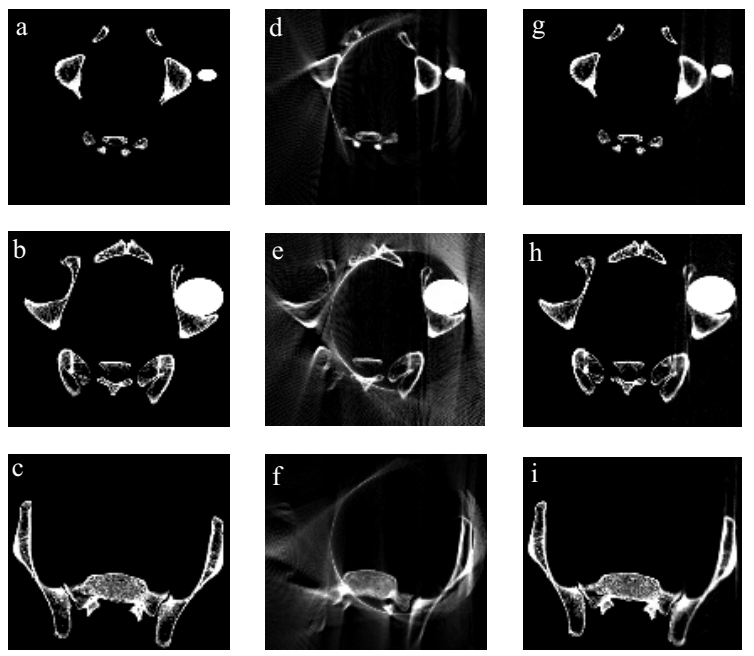

Fig. 4. The images in the first, second and third rows correspond to a slice below the mid-plane, a slice in the mid-plane and a slice above the mid-plane respectively. The left most column (panels a,b, and c) shows the modified CT for reference. Reconstructions using the FDK method (from $180^{\circ}$ ) are shown in panels d,e, and f. Reconstructions using our method (from $175^{\circ}$ ) are shown in panels $\mathrm{g}, \mathrm{h}$, and i. 
Our method exhibits (see Fig. 4) considerable improvement in the quality of reconstructions over the standard FDK algorithm. Truncation artifacts are visibly non-existent in comparison. While the central slice (Fig. 4h) is reconstructed perfectly, there is also considerable improvement in the quality of the other slices (Figs. $4 \mathrm{~g}$ and $4 \mathrm{i}$ ).

In the case of real experimental data, robust 2D-3D registration algorithms can be used to register the pre-operative CT scan with the inter-operative projections images. There is also a necessity to correct the x-ray energy differences between the two, so as to obtain consistent line integrals. Further improvement in efficiency (smaller angular sweep) of the source path can be attained by carefully planning the position of the iso-center of the $\mathrm{C}$-arm and the starting angle, thereby reducing scan-time and x-ray exposure.

\section{References}

1. H.K. Tuy. An inversion formula for cone-beam reconstruction. SIAM Journal of Applied Mathematics, 43:546-552, 1983.

2. J.X. Chen. A theoretical framework of regional cone-beam tomography. IEEE Transactions on Medical Imaging, 11:342-350, 1992.

3. L.A. Feldkamp, L.C. Davis, and J.W. Kress. Practical cone-beam algorithm. Optical Society of America, 1(6), June 1984.

4. A.C. Kak and Malcolm Slaney. Principles of Computerized Tomographic Imaging. Society of Industrial and Applied Mathematics, 2001.

5. Dennis L. Parker. Optimal short scan convolution reconstruction for fanbeam ct. Physics in Medicine and Biology, 9:254-257, 1982.

6. Frederic Noo, Michel Defrise, Rolf Clackdoyle, and Hiroyuki Kudo. Image reconstruction from fan-beam projections on less than a short-scan. Physics in Medicine and Biology, 47:2525-2546, July 2002.

7. Jens Müller-Merbach. Manual: Simulation of X-Ray Projections For Experimental 3D Tomography, 1996.

8. K. Wiesent, K. Barth, N. Navab, P. Durlak, T. Brunner, O. Schuetz, and W. Seissler. Enhanced 3-d-reconstruction algorithm for c-arm systems suitable for interventional procedures. IEEE Transactions on Medical Imaging (ITMI), 19(5):391-403, May 2000.

9. N. Navab, A. Bani-Hashemi, M. Nadar, K. Wiesent, P. Durlak, T. Brunner, K. Barth, and R. Graumann. 3d reconstruction from projection matrices in a c-arm based 3d-angiography system. First International Conference: Medical Imaging Computing and Computer-Assisted Intervention (MICCAI), October 1998.

10. N. Navab, M. Mitschke, and O. Schuetz. Camera augmented mobile c-arm (camc) application: 3d reconstruction using a low-cost mobile c-arm. Second International Conference: Medical Imaging Computing and Computer-Assisted Intervention (MICCAI), September 1999. 